\section{Aprendizaje del razonamiento clínico por reconocimiento de patrón, en seminarios de casos clínicos prototipos, por estudiantes de tercer año de medicina}

\author{
GUSTAVO MONTALDO L. ${ }^{1, a}$, PEDRO HERSKOVIC L. ${ }^{2}$
}

\section{Teaching of clinical reasoning to medical students using prototypical clinical cases}

\begin{abstract}
Background: Clinical reasoning is the most important competence in the training process of a physician. Aim: To develop a method for teaching clinical reasoning based on prototypes of clinical cases. Material and Methods: The study was conducted on sixty-four third year medical students. The study and control groups attended lectures and tutorial sessions with patients. The study group attended additionally discussion seminars of prototypical clinical cases. A clinical reasoning test was applied at the start and end of the learning period to both groups. At the end of the study, the opinions of students of the study group were collected in a focus group. Results: After the learning period, both groups significantly increased their clinical reasoning skills. However, the improvement in the study group was more than double than that of the control group. The absolute improvement in the study group was 30.9\%. Students interviewed in the focus group were unanimous in expressing their satisfaction in each and every aspect discussed. Conclusions: The teaching of clinical reasoning to third year medical students by means of pattern recognition in seminars with clinical cases improved significantly their skills.
\end{abstract}

(Rev Med Chile 2013; 141: 823-830).

Key words: Clinical Competence; Diagnosis differential; Decision Making; Education, medical, undergraduate.

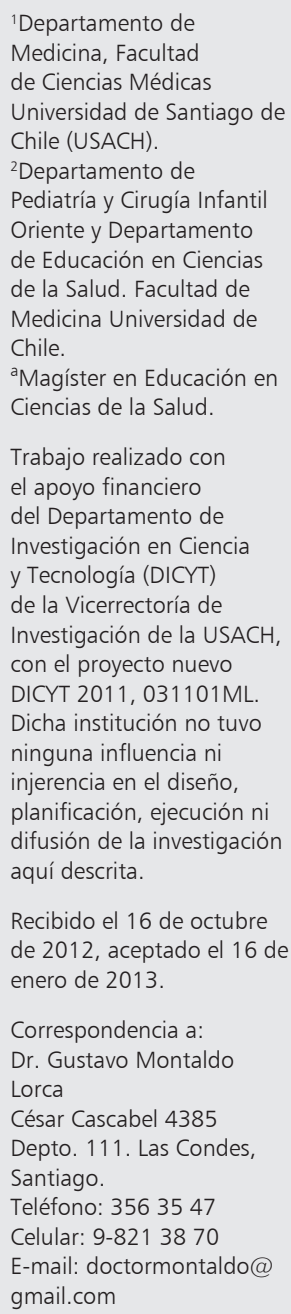

$\mathrm{E}$ 1 razonamiento clínico (RC) es el proceso cognitivo que le permite al médico elaborar un diagnóstico, tomar decisiones respecto del estudio y tratamiento, elaborar un pronóstico, y resolver el problema de salud del paciente ${ }^{1-3}$. Es la competencia transversal más importante en la formación de pregrado de un médico, y las escuelas de medicina se comprometen técnica y éticamente a su enseñanza, garantizando mínimos de cumplimiento ${ }^{4,5}$.

Variadas son las estrategias de razonamiento que pueden ponerse en juego ${ }^{6}$.

La estrategia por "reconocimiento de patrón" o raciocinio no analítico, se precipita de forma automática en el médico examinador, por la similitud que el caso presente tiene con casos previamente conocidos $^{7,8}$. Ello ocurre cuando el médico identifica o reconoce en el paciente, un determinado "patrón" o "guión de enfermedad". Es el método empleado por los médicos expertos, en la mayoría de los casos. ${ }^{9}$. De las estrategias diagnósticas conocidas, es ésta la que demuestra tener la más alta probabilidad de éxito diagnóstico ${ }^{10}$. En casos poco habituales, otras son las estrategias que se emplean.

El guión de enfermedad es la estructura cognitiva que tiene el clínico para almacenar y ordenar la información clínica relevante, que se enriquece con cada nuevo caso clínico que presente igual patología ${ }^{11,12}$. El guión permite al médico construir interpretaciones respecto de la situación clínica 
presente, con la consiguiente elaboración de una hipótesis diagnóstica ${ }^{13}$.

La estructura del guión está constituida por los factores predisponentes y las manifestaciones clínicas.

Estas últimas se expresan por la combinación de hechos semiológicos tanto anamnésticos como del examen físico cuya identificación en un nuevo paciente sugieren en el clínico, el diagnóstico de su condición patológica ${ }^{14}$.

Hasta hoy, el aprendizaje del razonamiento clínico se hace por modelaje del médico tutor a su aprendiz, durante la actividad clínico docente en sala con pacientes. El modelaje resulta una actividad docente no programada, intuitiva, no estandarizada ni estructurada. No existe un método sistemático que promueva dicho aprendizaje, como tampoco, un sistema de evaluación acorde.

Los alumnos y los médicos novicios demuestran en la práctica clínica insuficiencias en la elaboración de un razonamiento clínico satisfactorio ${ }^{15}$.

La consecuencia es la elaboración incorrecta o no oportuna del diagnóstico clínico, y en consecuencia, la inadecuada toma de decisiones.

Sin embargo, dicha estrategia de razonamiento se puede formar en los estudiantes y médicos novicios, gracias al aprendizaje de los guiones de enfermedad en un caso clínico prototipo ${ }^{16,17}$.

Para ello, se diseña un método de enseñanza teórico en aula, que consiste en seminarios de casos clínicos prototipos de las patologías más prevalentes en el adulto en Chile, susceptibles de ser diagnosticadas por reconocimiento de patrón.

Dichos guiones de enfermedad (patrones) son susceptibles de ser identificados, analizados, confrontados y enriquecidos con lo aprendido por el estudiante con su docente, al lado del enfermo.

De igual modo, y toda vez que se justifique, se contempla también la enseñanza de los guiones de las variantes clínicas menos frecuentes de acuerdo a patología ${ }^{14}$. Así, imitamos la fortaleza del conocimiento diverso del experto, al elaborar su diagnóstico en casos clínicos con presentación "atípica"18.

Nuestro estudio tiene como objetivo general, la promoción y mejoramiento del aprendizaje del razonamiento clínico. Los propósitos específicos son la elaboración de un método de enseñanza del razonamiento clínico basado en seminarios de casos clínicos prototipos, su aplicación, y la evaluación de dicho aprendizaje.
Aprendizaje, que imita el modo de diagnosticar propio de los expertos ${ }^{19-21}$.

\section{Material y Método}

\section{Población en estudio}

Sesenta y cuatro estudiantes del tercer año de medicina de la Universidad de Santiago de Chile (USACH), que realizan su práctica clínica de Semiología y Medicina Interna en hospital, desde el 1 de marzo al 31 de octubre de 2011.

Estudio cuantitativo: La muestra corresponde a 27 estudiantes asignados aleatoriamente a realizar su práctica clínica en el hospital Barros Luco (grupo control), y 37 estudiantes, en el hospital San José (grupo en estudio).

Estudio cualitativo: La muestra corresponde a nueve estudiantes seleccionados aleatoriamente, del grupo en estudio.

\section{Método}

2.1. Estudio cuantitativo, diseñado para evaluar logros de aprendizaje del razonamiento clínico (calidad de los diagnósticos sindromático, etiológico y diferencial; y su fundamentación por identificación del patrón de enfermedad).

Se emplea un diseño pre prueba-post prueba y grupo control ${ }^{22}$. El análisis de datos se hace mediante la aplicación del test U Mann Whitney para eventos pareados e independientes ${ }^{22}$.

2.2. Estudio cualitativo, terminado el estudio y con el propósito de conocer la experiencia, opiniones y percepciones de los estudiantes respecto del método empleado, se realiza un Grupo Focal ${ }^{23}$.

\section{Instrumentos}

\subsection{La intervención educacional}

a) En el grupo control: la intervención educacional consiste en la docencia teórico práctica tradicional, que incluye: $108 \mathrm{~h}$ de clases expositivas, y $450 \mathrm{~h}$ de docencia tutorial al lado del enfermo.

b) En el grupo en estudio, la intervención consistió en la docencia tradicional: $108 \mathrm{~h}$ de clases expositivas y $380 \mathrm{~h}$ de docencia tutorial; más $72 \mathrm{~h}$ de Seminarios de Casos Clínicos Prototipo. Se realizaron 36 seminarios de $2 \mathrm{~h}$ de duración, durante 6 meses.

3.2. Los casos clínicos (Anexo 1). Se construyen ochenta casos clínicos que se usan como prototipos de síndromes y de patologías cardiológicas y pulmonares típicas y atípicas. La estructura de 
cada caso clínico incluye los guiones de enfermedad (illness scripts) destacados "en negrita". A continuación, se explicitan los signos del examen físico que incluyen: signos vitales y signos destacados del examen físico general y del examen físico segmentario. También, y si el análisis clínico lo amerita, se incluyen los resultados de exámenes de laboratorio, electrocardiograma e imágenes.

3.3. La prueba de razonamiento clínico (PRC). Anexo 2. Se construye el instrumento de evaluación del logro de aprendizajes del razonamiento clínico, constituido por diez casos clínicos proble$\mathrm{ma}^{16}$. Cada caso contiene cinco preguntas abiertas. La evaluación se hace asignando un punto por respuesta correcta. El puntaje teórico máximo es de 50 puntos. El rendimiento se expresa como el porcentaje de respuestas correctas.

\subsection{Validación por expertos.}

3.4.1. Un grupo de cinco expertos, todos médicos clínicos y académicos de la Universidad de Chile $^{2}$ y de la USACH ${ }^{3}$, validan:

a) Los 80 casos clínicos contenidos en los Seminarios de Casos Clínicos, Anexo 1.

b) Los 10 casos clínicos contenidos en la Prueba de Razonamiento Clínico, Anexo 2.

3.4.2. Un grupo de ocho médicos post becados de Medicina Interna de la USACH validan las respuestas a cada pregunta de la prueba, al someterse a la PRC en iguales condiciones que los estudiantes de tercer año.

3.4.3. Un grupo de cuatro expertas en educación, académicas de la Universidad de Chile, validan el cuestionario para el grupo focal.

\section{Aspectos éticos}

a) El proyecto se presenta al director del Departamento de Medicina, y al director de la Escuela de Medicina de la USACH, quienes dan su aprobación para la implementación de la investigación educacional.

b) Consentimiento informado. Los estudiantes lo suscriben libremente, previo a rendir la PRC, pre y post intervención educacional.

\section{Resultados}

\section{Estudio cuantitativo}

La PRC se realizó en ambos grupos, antes y después de la intervención educacional: PRC 1 (marzo) y PRC 2 (octubre).
1. Evaluación del logro de Aprendizajes, en grupo control y grupo en estudio.

1.1. Logro de aprendizajes en grupo control. Tabla 1.

En este grupo, la media de rendimiento en la PRC 1 previa a la docencia tradicional, fue de $4,29 \%$. En la PRC 2, fue de 32,03\% ( $\mathrm{p}=0,000)$.

Existe una diferencia significativa entre ambos rendimientos.

1.2. Logro de aprendizajes en el grupo en estudio. Tabla 2.

En el grupo en estudio, la media de rendimiento en la PRC 1 fue de 3,35\%. En la PRC 2, fue de $59,52 \%(p=0,000)$. Existe una diferencia significativa entre ambos rendimientos.

2. Comparación de logro de aprendizajes entre grupo en estudio, y grupo control. En cada grupo, se procedió a sustraer el rendimiento de la PRC1 de la PRC 2, para cada estudiante. Se obtuvo un $\Delta$ para cada alumno. Se sumaron estos $\Delta$, y el total se dividió por el número de alumnos. Tabla 3.

El incremento de rendimiento en el grupo control, después de la docencia tradicional, fue de $24,64 \%$. En el grupo en estudio, fue de $55,77 \%$ $(\mathrm{p}=0,000)$.

Tabla 1. Logro de aprendizajes en grupo control

\begin{tabular}{|ccc|}
\hline PRC & Media (\%) & $\mathbf{p}=$ \\
\hline 1 & 4,29 & \\
2 & 32,03 & 0,000 \\
\hline
\end{tabular}

PRC: prueba de razonamiento clínico.

Tabla 2. Logro de Aprendizajes en grupo en estudio

\begin{tabular}{|ccc|}
\hline PRC & Media (\%) & $\mathbf{p}=$ \\
\hline 1 & 3,35 & \\
2 & 59,92 & 0,000 \\
\hline
\end{tabular}

PRC: prueba de razonamiento clínico.

Tabla 3. Logro de aprendizajes comparado, de grupo control y en estudio

\begin{tabular}{|lcl|}
\hline Grupo & $\frac{\Sigma \Delta \text { (PRC 2 - PRC 1) }}{\mathbf{n}}$ & $\mathbf{p}=$ \\
\cline { 2 - 3 } Control & 24,6 & \\
Estudio & 55,8 & 0,000 \\
\hline
\end{tabular}


Ello significa que hay una diferencia significativa entre los incrementos de rendimientos del grupo en estudio, respecto del grupo control (es significativo todo valor de $\mathrm{p} \leq \mathrm{a} 0,05)$.

\section{Estudio cualitativo}

Categorías. Las categorías definidas para el grupo focal fueron: aprendizaje, metodología, evaluación, condiciones y sugerencias. La categoría emergente del estudio fue la interacción.

Se elaboró un cuestionario que pretende dar respuesta a las categorías así enunciadas. Anexo 3.

\section{Aprendizajes}

a) Todos los estudiantes le asignaron importancia al método, porque pueden: "comprender, elaborar y desarrollar el razonamiento clínico", "identificar un patrón y elaborar diagnóstico", "reforzar, jerarquizar y aplicar lo aprendido en sus pacientes"; b) Todos opinaron que "el método sólo muestra ventajas, y contribuye significativamente al aprendizaje clínico"; c) La retroalimentación del docente les permite a los estudiantes: "desarrollar mejores aprendizajes e incorporar nuevas formas de análisis". "elaborar un juicio crítico frente a un problema clínico", "evaluar gradualmente sus propios aprendizajes durante el proceso".

\section{Metodología}

a) Todos los estudiantes "toman decisiones referidas al estudio del paciente: qué exámenes solicitar y su fundamento". También "manifiestan su interés porque el método sea incorporado en el programa del curso de semiología y medicina interna de nuestra escuela".

b) Agregan: "es el mejor método para consolidar los conocimientos clínicos" y "nos permite formar un juicio crítico respecto del paciente".

\section{Evaluación}

a) Todos coincidieron en afirmar que: "las evaluaciones escritas de la asignatura no reflejan ni evalúan los aprendizajes del razonamiento clínico hecho en seminarios".

b) Respecto a las evaluaciones realizadas por sus tutores en sala con pacientes, afirmaron: "pudimos emplear el razonamiento clínico con éxito, elaborar los diagnósticos sindromáticos y etiológicos correctos y demostrar nuestra comprensión del enfermo".

\section{Condiciones de Aprendizaje}

a) Respecto a las dos horas de duración del seminario, "era un tiempo que se aprovechaba de principio a fin en aprendizaje".

b) En cuanto a la estructura del seminario: "muy buena, en especial la presentación del caso, los comentarios y los consejos dados por el profesor".

c) En cuanto al número de estudiantes: "en pequeño grupo, es excelente".

d) Respecto del espacio físico: a todos, les pareció "adecuado".

\section{Sugerencias}

- "Aumentar la frecuencia de los seminarios".

- "Incorporar imágenes de ECG, radiografías y algunos procedimientos".

\section{Interacción}

a) "El método nos ha permitido relacionarnos de manera segura y fundada con los médicos tutores y los propios pacientes".

b) La unanimidad reconoció la importancia de la interacción y retroalimentación con el profesor: "él vierte su experiencia clínica personal y subraya las variantes clínicas"; "nos ayuda a fijar conceptos, nos motiva y promueve nuestra reflexión".

\section{Discusión}

$\mathrm{Al}$ ingresar al tercer año, y previo a la intervención educacional, las medias de rendimiento en ambos grupos son muy bajas, y no existen diferencias entre ambos: $4,29 \%$ y $3,35 \%$, respectivamente $(\mathrm{p}>0,05)$.

Después de la intervención educacional, los estudiantes de ambos grupos muestran incrementos significativos en el logro de aprendizaje del razonamiento clínico: $32,03 \%$ y 59,92\% respectivamente $(\mathrm{p}=0,000)$.

Cuando se comparó el incremento de rendimiento habido entre el grupo control y el grupo en estudio, este fue de $24,64 \%$ para el primero, $y$ de $55,57 \%$ para el segundo. El incremento de rendimiento en el grupo en estudio, más que el doble del grupo control.

Michel Groves y cols. también obtienen logros significativos con el método, aunque menores:14\% superior en el grupo en estudio, respecto del grupo 
Aprendizaje del razonamiento clínico - G. Montaldo et al

control ( $43 \%$ vs $29 \%)^{16}$. En nuestro estudio es de $55,7 \%$ vs $24,64 \%$ respectivamente. Esto es, un incremento absoluto de 30,9\%.

El menor incremento obtenido por los autores australianos puede atribuirse a lo breve de la experiencia, aspecto que es reconocido por los autores y estudiantes del grupo en estudio.

Los buenos resultados obtenidos por nosotros, comparados con la docencia tradicional, están relacionados a la innovación metodológica implementada durante el período en estudio. Las razones que pueden ayudar a entender estos resultados se encuentran, en nuestra opinión, en la experiencia de aprendizajes vivida por los estudiantes en este contexto, y que se expresan en el grupo focal.

La información recabada en el grupo focal expresa la satisfacción de los estudiantes por los variados aspectos estudiados en las diferentes categorías.

Un aspecto relevante es no sólo el relativo al aprendizaje y desarrollo del razonamiento clínico que experimentan, sino que, a la posibilidad de aplicarlo en pacientes reales, como también, en casos clínicos problema.

Los estudiantes manifiestan haber ganado en juicio crítico y confianza para expresar, fundamentar y sostener sus opiniones con pares, tutores y pacientes.

El desarrollo de la competencia del razonamiento clínico a nivel de tercer año, es el de elaborar los diagnósticos sindromático, etiológico y diferencial. El resto de su aprendizaje (toma de decisiones y elaboración de un pronóstico) está previsto para ser desarrollado en cuarto año, y por cierto, continuado en el internado de medicina.

La metodología de seminarios de casos clínicos prototipos tiene como propósito generar en los estudiantes redes de asociación e integración, relaciones de orden y jerarquización conceptuales, que les permitan frente a un enfermo, elaborar un diagnóstico eficiente y oportuno.

El método de reconocimiento de patrón en casos clínicos prototipos -como intervención educacional complementaria de la actividad clínica en sala-incrementó significativamente el aprendizaje del razonamiento clínico, en los estudiantes de tercer año de la Escuela de Medicina de la Universidad de Santiago de Chile.

La opinión unánime vertida por los estudiantes respecto de las categorías evaluadas en relación al método, demuestran el valor y la importancia que ellos le asignan. Los estudiantes son capaces de reconocer patrones de manera correcta, y elaborar los diagnósticos correspondientes.

Aunque los resultados no son susceptibles de generalización, ni nos permiten hacer una proyección respecto del desempeño de estos estudiantes en su desempeño profesional futuro, los resultados de la metodología son muy promisorios. La experiencia constituye fundamento y antecedente sólido para ser reproducida en nuestra propia escuela, como también en otras escuelas de medicina del país.

No existe en nuestra realidad, local ni mundial, antecedentes documentados que den cuenta del crecimiento y progresión de esta competencia.

Esta investigación aporta información en tal sentido, con ocasión de la introducción de un método que pretende mejorar el aprendizaje del razonamiento clínico en estudiantes que carecen de toda experiencia clínica previa.

La metodología parece promover en los estudiantes la construcción de redes de asociación e integración, de conocimiento ordenado, profundo y diverso, de aprendizajes con sentido. De igual manera, contribuye a generar en ellos un pensamiento de calidad: crítico, creativo y metacognitivo.

El razonamiento clínico constituye el principal método de interacción clínica en el servicio profesional y ético al enfermo, y supone conocimiento, reflexión y acción. Esta investigación pretende ser un aporte al conocimiento y desarrollo de esta competencia fundamental en la formación médica de nuestro tiempo. 
Anexo 1. Caso Clínico (incluye el guión de enfermedad, en negrita)

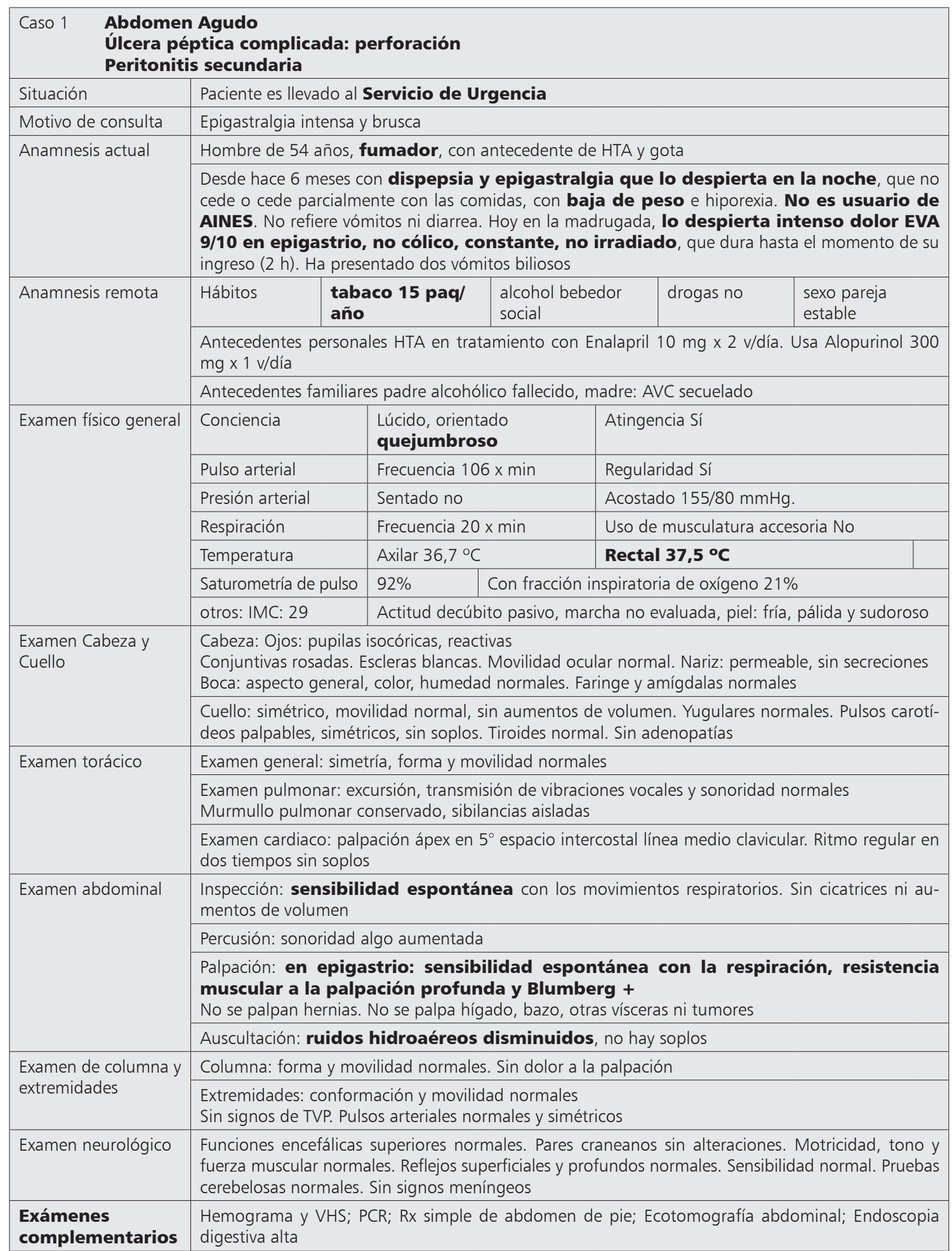




\section{Anexo 2. Prueba de Razonamiento Clínico: caso problema (incluye 5 preguntas)}

Mujer de 54 años, portadora de HTA y DM2 desde hace 15 años. En tratamiento irregular. Usuaria habitual de broncodilatadores. Hoy en la madrugada se despierta en forma brusca con intensa disnea, tos y expectoración que la obligan a incorporarse de la cama. Con escasa respuesta a Salbutamol. No refiere dolor torácico ni síncope.

Lúcida, orientada, angustiada. Pulso: 100/min reg; P. Art.: 170/120 mmHg; Resp: 26/min; to ax: 36,9 ${ }^{\circ} \mathrm{C}$, IMC: 33 . Ortopneica, pálida y sudorosa. Ex pulmonar: transmisión de vibraciones vocales normales y sonoridad normales. Murmullo pulmonar conservado, con abundantes roncus y sibilancias en ambos campos.

Sin estertores ni crépitos. Corazón: ápex en $6^{\circ}$ EIC por fuera LMCl. Ritmo en 2 tiempos, sin soplos. Resto del examen físico dentro de rango normal.

1. ¿Cuál es el diagnóstico Sindromático?

2. ¿Cuál es el diagnóstico Etiológico más probable?

3. Haga una lista con los hechos clínicos que fundamentan su diagnóstico etiológico (+) y aquellos que se oponen (-), y asigne a cada uno, el nivel de importancia que corresponda.

\begin{tabular}{|l|l|l}
\hline Antecedentes clínicos síntomas y/o signos & Apoya (+) Opone (-) & $\begin{array}{l}\text { Importancia: } 1 \text { poca; } \\
2 \text { mediana; } 3 \text { mucha }\end{array}$
\end{tabular}

4. ¿Cuál es el principal diagnóstico Diferencial?

5. Haga una lista con los hechos clínicos que fundamentan su diagnóstico diferencial (+) y aquellos que se oponen (-), y asigne a cada uno, el nivel de importancia que corresponda

\begin{tabular}{|l|l|l}
\hline Antecedentes clínicos síntomas y/o signos & Apoya (+) se Opone (-) & Importancia: 1 poca;
\end{tabular}

2 mediana; 3 mucha

\section{Anexo 3. Cuestionario grupo focal}

1. ¿Tuvo importancia el método de seminarios de casos clínicos, en su aprendizaje clínico?

2. ¿Pudo emplear el método de reconocimiento de patrón en su práctica clínica?

3. Los diagnósticos elaborados con el método, ¿lo habilitaron para la toma de decisiones?

4. ¿El método le sirvió para enfrentar la evaluación del curso?

5. ¿Qué ventajas y desventajas tiene el método de seminarios, respecto a la clase expositiva?

6. ¿Qué importancia le asigna a la retroalimentación dada por el profesor?

7. ¿Qué opiniones le merecen las condiciones de aprendizaje?

8. ¿Qué sugerencias tiene usted para mejorar el método de seminarios de casos clínicos?

\section{Referencias}

1. Audétat MC. Méthodes et outil pour soutenir et stimuler un raisonnement clinique efficace. Canadian family physician 2010; 56 (3): 294-6.

2. Higgs J. Clinical reasoning in the health professions (third edition). Butterworth Elsevier. 2008; Chapter 1: p. 3-7.

3. Norman G. Building on experience. The development of clinical reasoning. New England Journal of Medicine 2006; 355 (21): 2251-2.

4. Croskerry P. A universal model of diagnostic reasoning. Academic Medicine 2009; 84: 1022-8.
5. Norman G. Reaserch in clinical reasoning: past history and current trends. Medical Education 2005; 39: 418-27.

6. Norman G. Doggie diagnosis, diagnostic reasoning strategies: an alternative view. Medical Education 2003; 37: 676-7.

7. Norman G. The non analytical basis of clinical reasoning. Advances in health sciences education 1997; 2: 173-84.

8. Regher G. Effect of processing strategy on diagnostic skill in dermatology. Academic Medicine 1994; 69 (10): s34-6.

9. Norman G. Expertise in visual diagnosis: a review of the literature. Academic Medicine 1992; 67 (10): s78-s83. 
10. Coderre S. Diagnostic reasoning strategies and diagnostic success. Medical Education 2003; 37: 695-703.

11. Schmidt HG. A cognitive perspective on medical expertise: theory and implications. Academic Medicine 1990; 65 (10): 611-21.

12. Schmidt HG. How expertise develops in medicine: knowledge encapsulation and illness script formation. Medical Education 2007; 41: 1133-9.

13. Charlin B. Scripts and medical diagnostic knowledge: theory and applications for clinical reasoning instruction and reaserch. Academic Medicine 2000; 75 (2): 182-90.

14. Bowen J. Educational strategies to promote clinical diagnostic reasoning. New England Journal of Medicine 2006; 355 (21): 2217-25.

15. Kassirer J. Learning clinical reasoning (second edition). Philadelphia: Lippincott Williams \& Wilkins. 2010; Chapter 11: p. 123-33.

16. Groves M. Using illness scripts to teach clinical reasoning skills to medical students. Family Medicine 2010; 42 (4): 255-61.

17. Norman G. Non analytical models of clinical reasoning: the role of experience. Medical Education 2007; 41: 1140-5.

18. Ericsson KA. An expert performance perspective of reaserch on medical expertise: the study of clinical performance. Medical Education 2007; 41: 1124-30.

19. Kassirer J. Teaching clinical reasoning: case based and coached. Academic Medicine 2010; 85 (7): 1118-24.

20. Mandin H. Helping students learn to think like experts when solving clinical problems. Academic Medicine 1997; 72 (3): 173-9.

21. Quinton A. L' enseignement du raisonnement clinique: les stratégies diagnostique et thérapeutique, l' établissement du prognostic. Diplôme d' université, de pédagogie en sciences de la santé à distance: 1-14. Université de Bordeaux, France. 2007. www.crame.ubordeaux2.fr/ pdf/ enseignementraisonnementclinique.pdf (acceso el 19.07.2013).

22. Sidney S. Estadística no paramétrica, aplicada a las ciencias de la conducta. Ed. Trillas. 1990; p. 143.

23. Hernández R. Metodología de la Investigación. México, Mc Graw-Hill Interamericana. Recolección y análisis de datos cualitativos. 2010; 406-88. 